\title{
Solubility Studies and Growth of 4-aminobenzophenone Single Crystal: a Potential Organic NLO Material
}

\author{
V. Natarajan \\ Department of Physics, \\ Dr. Sivanthi Aditanar College of Engineering, \\ Tiruchendur, India
}

\author{
S. Usharani \\ Department of Physics, \\ Dr.Sivanthi Aditanar College of Engineering, \\ Tiruchendur, India
}

\author{
M. Arivanandhan \\ Research Institute of \\ Electronics, \\ Shizuoka University, \\ Hamamatsu, Japan
}

\author{
P. Anandan \\ Research Institute of \\ Electronics, \\ Shizuoka University, \\ Hamamatsu, Japan
}

\begin{abstract}
The solubility and growth aspects of 4-aminobenzophenone (4-ABP) was investigated with an intention to grow bulk and good quality crystal. The solubility of 4-ABP in various organic solvents such as ethyl acetate, ethanol and O-xylene were studied. From the investigation, it was realized that the 4-ABP material has highest solubility in ethyl acetate whereas it has the lowest solubility in pure Oxylene solvent. Single crystals of 4-ABP were grown by solution growth using ethanol as a growth medium which has the moderate solubility when compared to other solvents. The structural and optical properties of the grown 4-ABP samples were studied. The lattice parameters of the 4-ABP were calculated using X-ray diffraction analysis which confirmed the monoclinic structure of the grown material. The optical transmission study showed that the $4-\mathrm{ABP}$ has about $70 \%$ of transparency in the visible and near infrared region
\end{abstract}

\section{INTRODUCTION}

Organic materials are promising for nonlinear optical (NLO) applications because of their high NLO efficiency and fast response than their inorganic counter parts. Benzophenone and its derivatives are potential organic NLO material with good optical, mechanical properties, and non hygroscopic nature [1, 2]. Moreover, the derivatives of benzophenone are attracted by many researchers due to their large NLO susceptibilities and short cut-off wavelengths up to deep UV region $(<400 \mathrm{~nm})[3]$. Among the benzophenone derivatives, 4-amino benzophenone (4-ABP) is the most significant one with the second harmonic generation (SHG) efficiency of 360 times that of Ammonium dihydrogen Phosphate (ADP) [3, 4]. The 4-ABP crystal belongs to the monoclinic crystal system with space group P21 and cell parameters $\mathrm{a}=12.036 \AA, \mathrm{b}=5.450 \AA, \mathrm{c}=8.299 \AA$, and $\beta=$ $97.841^{\circ}$. Even though several reports are available on the growth aspect of 4-ABP, growth of high quality, inclusion free bulk crystal of 4-ABP is still remaining as a difficult task due to its growth related issues. Moreover, it is difficult to grow 4-ABP single crystal from solution, because it is readily photo-oxidized [5] in solution which results pink colored impurity and it can be easily incorporated into growth sectors of the transparent crystal [5]. For the growth of 4-ABP, several organic solvents such as ethanol, methanol, dimethyl formamide (DMF) were used as the growth medium in the literature [5, 6]. Pan et al. [6] investigated the effect of ethanol, methanol and DMF solvents on the growth of 4-ABP crystals. Recently, the 4-ABP single crystal with dimensions of $30 \times 9 \times 6 \mathrm{~mm}^{3}$ was grown by using ethyl acetate as a solvent by Natarajan et al [7]. However, lots of inclusions were found in the grown crystals from ethyl acetate solution which resulted low quality crystals with less transparency.

In this present investigation, the solubility of 4-ABP was measured in three different solvent at ambient temperature and relatively inclusion free 4-ABP crystal was grown by using ethanol as a growth medium. The XRD and optical transmission studies were carried out on the grown crystals to analyze the structural and optical properties of the 4-ABP.

\section{SOLUBILITY AND CRYSTAL GROWTH OF 4-ABP.}

The commercially purchased 4-ABP material was purified by recrystallization process using ethanol. In the solution growth process, the solvent can influence the morphology of the crystal due to its interaction with the surfaces of the growing crystal. Therefore, the selection of suitable 
solvent for crystal growth is more important in solution growth. The solubility of the 4-ABP in various organic solvents viz., the ethyl acetate, the ethanol, and o-xylene was measured at ambient temperature. From the investigation, it was found that the 4ABP material has highest solubility in ethyl acetate $(17 \mathrm{~g} / 100$ $\mathrm{ml})$ whereas it has the lowest solubility in pure O-xylene (1 $\mathrm{g} / 100 \mathrm{ml})$. But, it has moderate solubility in ethanol $(10 \mathrm{~g} / 100$ $\mathrm{ml}$ ). In general, if the solubility is too high, it is difficult to grow good quality large size crystal as it is difficult to control the number of nucleation. On the other hand, too low solubility is also not suitable to grow crystal with reasonable size due to low availability of solute molecules. Therefore, ethanol was chosen as a growth medium for the present work and the saturated solutions of 4-ABP was prepared by dissolving the refined source material into ethanol. The prepared saturated solutions were homogenized by continuous mild stirring of the solutions using a magnetic stirrer by maintain the temperature of the solution. Then, the near saturated solutions were transferred into a crystallizer and covered by a perforated polyethylene sheet for controlled evaporation at room temperature. After the growth period of 7 days, we obtained the relatively inclusion free and platelet crystals from the growth solution prepared by using ethanol. The photograph of the harvested crystal from ethanol is shown in Fig.1.

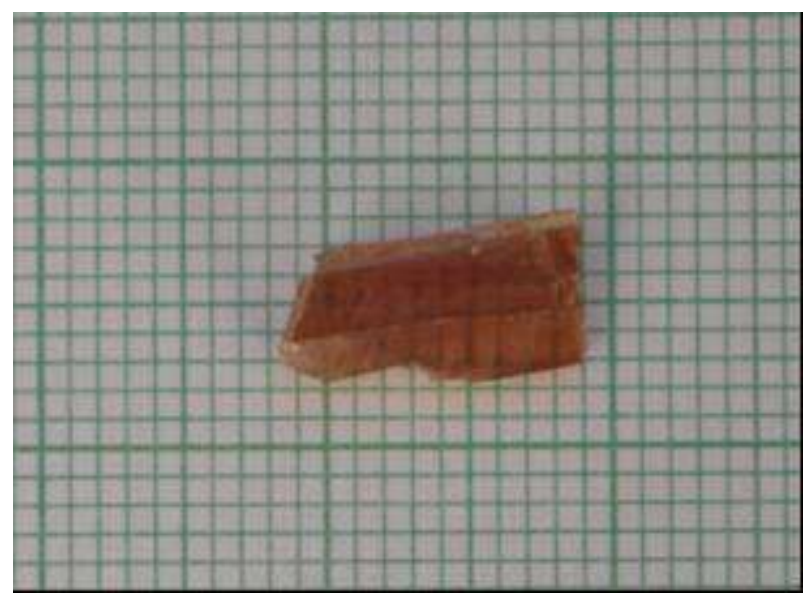

Figure 1: Photograph of the 4-ABP crystal grown from ethanol.

\section{RESULTS AND DISCUSSION}

At the initial stage the growth solution was looked as pale yellow color and when the time increases, the solution turned to dark yellow in color. This color variation is possibly due to photo oxidation of the growth solution [6]. Moreover, the grown crystal also looks like dark yellow in color. However, the crystal is relatively inclusion free as can be seen in the Fig.1, when compared to our previous crystal grown in ethyl acetate solution [7]. Low growth rate of the crystal (compared to crystal grown from ethyl acetate) is the possible reason for the low solvent inclusion. Further investigation is going on to improve the quality of the crystal by mixed solvent approach and the outcome will be published elsewhere. The structural properties of the grown crystals were studied by X-ray diffraction (XRD) analysis using Rigaku X-ray diffractometer. Optical transmission study was performed at UV Vis-NIR region to determine the range of transmission/absorption of the grown crystals.

\subsection{X-ray diffraction analysis of the grown samples}

The recorded X-ray diffraction spectrum for the fine powdered 4-ABP sample is shown in Fig. 2. The sharp and strong diffraction peaks of the grown samples illustrate the crystalline quality of the samples. The diffraction peaks of the XRD spectra were indexed and thus lattice parameters of the crystal were calculated using the crystallographic equation. The 4-ABP crystal belongs to monoclinic crystal system with P21 space group. The calculated lattice parameters of 4-ABP sample are $\mathrm{a}=12.35 \AA, \mathrm{b}=5.59 \AA, \mathrm{c}=8.487 \AA$ and $\beta=97.41^{\circ}$ and the calculated parameters were well agreed with the reported values [4].

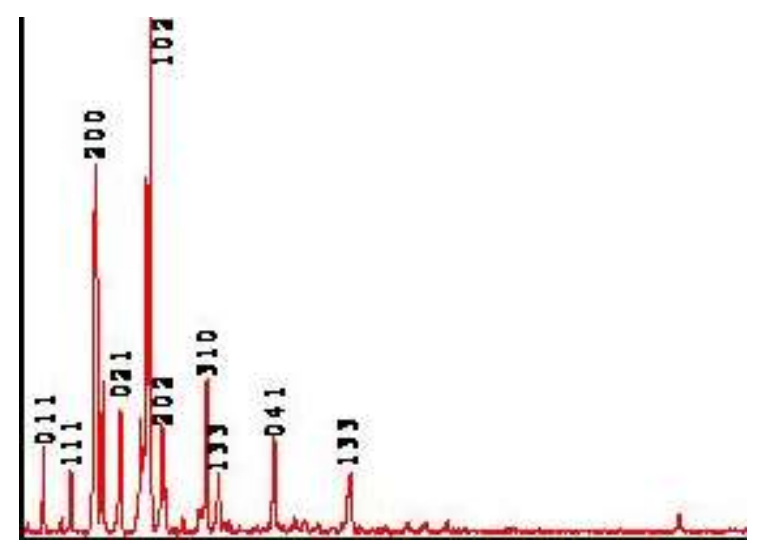

Figure 2: X-ray diffraction spectrum for the fine powdered 4$\mathrm{ABP}$.

\section{2 optical transmission studies}

Figure 3 shows the optical transmission spectrum of 4-ABP crystal. The polished crystal used for the optical studies had the thickness of about $1 \mathrm{~mm}$. The dark yellow colored 4-ABP crystal grown from the ethanol medium shows the transparency of about $70 \%$ in the visible and NIR region. The cut off wavelength of 4-ABP was observed at about $400 \mathrm{~nm}$. From the result, it is obvious that the 4-ABP sample is suitable for green laser generation. 


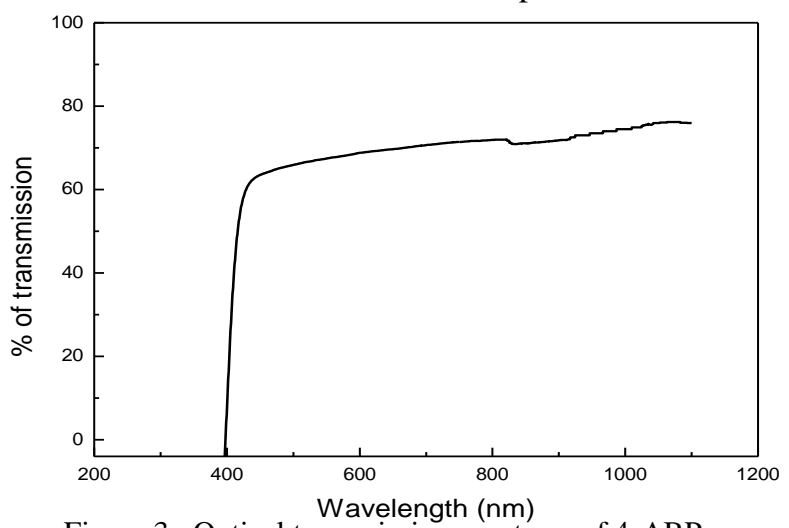

Figure 3: Optical transmission spectrum of 4-ABP.

\section{CONCLUSION}

Single crystals of 4-ABP were grown by solution growth using ethanol as a growth medium. The grown crystals were relatively inclusion free when compared to crystals grown from other solvents. The growth aspects of the crystals were analysed based on solute-solvent interactions. The structural and optical properties of the samples were investigated. It was confirmed that the 4-ABP was crystallized with monoclinic crystal system from the X-ray diffraction analysis. The 4-ABP crystal had relatively better transparency in the visible region with cut off wavelength of $400 \mathrm{~nm}$.

\section{REFERENCES}

1. Cockorham, P., Frazier, C.C., Guha, S., Chauchard, E.A., Appl. Phys. B53 (1991) 275.

2. Arivanandhan, M., Sankaranarayanan, K., Ramamoorthy, K., Sanjeeviraja C., Ramasamy, P., Opt. Mater. 27 (2005) 1864.

3. Frazier, C.C., Cockorham, P., Chauchard, E.A., Lee, C.H. , J. of Opt. Soc. of America B 4 (1987) 1899.

4. Jiang, M. Fang, Q. Advan. Mater. 11 (1999) 1147.

5. Cohen, S.G., Saltzman, M.D., Guttenplan, J.B., Tetra. Lett. 49 (1969) 4321.

6. Pan, F., Sherwood, J.N., Simpson, G.S. J. Mater. Chem. 7 (1997) 1383.

7. Natarajan, V. Omprakash, M. Arivanandhan, M., .and Hayakawa, Y., Mat. Chem. and Phys. 141 (2013) 160. 